\title{
Fatal infections in andrology. Atypical clinical presentation of a Fournier's disease
}

\author{
Valerio Olivieri ${ }^{1}$, Gabriele Ruggiero ${ }^{1,2}$, Danilo Abate ${ }^{2}$, Nicoletta Serra ${ }^{2}$, Valentina Fortunati ${ }^{3}$, \\ Daniele Griffa $^{1,2}$, Flavio Forte ${ }^{4}$, Emanuele Corongiu ${ }^{4}$ \\ ${ }^{1}$ Division of Urology, Ivrea civil Hospital (ASL TO4), Ivrea (Turin), Italy; \\ ${ }^{2}$ Division of Urology, Ciriè Hospital (ASLTO4), Ciriè (Turin), Italy; \\ ${ }^{3}$ Division of Pathology, Hospital "Policlinico of Tor Vergata", Rome, Italy; \\ ${ }^{4}$ Division of Urology, Hospital "Madre Giuseppina Vannini", Rome, Italy.
}

\begin{abstract}
Summary Background: Fournier disease (FD) is a worrisome infection of genital area caused by $a$ polimicrobial infection and characterized by a rapid progression to necrosis. Scrotum, perineum and lower abdomen represent the primary sites of origin. Clinical presentation and laboratory strongly suggest $F D$, but if not precociously diagnosed, it may quickly evolve into septic syndrome and patient's death. Case report: A 62 years old Caucasian male presented for fever and penile gross oedema recently occurred. No history of previous urinary tract infection, hematuria or genital trauma was referred. He did not complain any storage or voiding low urinary tract symptom (LUTS); no foci of infection in genitoperineal area was observed nor urethral discharge.

The ultrasound (US) revealed a disomogeneous broad thickening of subcutaneous tissues with increased vascularity on Color-Doppler. When the penis was manipulated in order to reduce oedema, retract foreskin and evaluate the glans, clinical parametres rapidly worsened and the patient developed a septic shock with blood pressure falling down, dyspnoea and tachyarrhythmia, and he was fastly sent to Intensive Care Unit where it has been hemodynamically stabilized and subjected to antibiotic therapy. Considering the clinical absence of gangrene's foci, we opted for a conservative treatment by maintaining bladder catheter and drug therapy.
\end{abstract}

KEY WORDS: Andrology; Fournier's disease; Atypical clinical presentation; Sepsis.

Submitted 2 March 2020; Accepted 15 March 2020

\section{INTRODUCTION}

Fournier's disease (FD) is a worrisome of genital area caused by a polimicrobial infection and characterized by a rapid progression to necrosis (1). It is a rare clinical condition accounting for 1.6 cases per 100.000 and represent the $0.01 \%$ to $0.06 \%$ of all the urological emergency (2). Elderly and defeated patients are usually involved. Scrotum, perineum and lower abdomen represent the primary sites of origin. It is characterized by a high rate of mortality since it quickly evolves into septic shock and patient's death. Hence, whenever misdiagnosed or not promptly treated, it may result in a fatal infection. Clinical presentation and laboratory strongly suggest FD. Atypical presentation has been also described in literature 1: if not precociously diagnosed, it may quickly evolve into septic syndrome and patient's death. We report the case of an adult male with uncommon clinical presentation. While opportunely describing this case, we also reviewed the literature about atypical FD.

\section{Case report}

A 62 years old Caucasian male presented for fever and penile gross oedema recently occurred. He was a smoker but familiarity for penile cancer was ruled out on anamnesis. No history of previous urinary tract infection, haematuria or genital trauma was referred. Additionally, he denied any sexual intercourse in the last four weeks. He was affected by metabolic syndrome and diabetes mellitus actually on medication with oral hypoglycemic agents. Dipstick urine confirmed glycosuria but was negative for urinary infection. Blood examinations were not available. He did not complain any storage or voiding low urinary tract symptom (LUTS); no foci of infection in genitoperineal area was observed nor urethral discharge. On physical examination the patient was febrile. On local examination inguinal lymph nodes were negative; there was no erythema of the scrotum and the testis were normal. Rectal exploration was unremarkable too with a soft enlarged painless prostate. However, the penis presented with an asymptomatic massive oedema extended from pubis to foreskin (Figure 1); it was also warm but painless and with a normal skin appearance. The ultrasound examination of the abdomen and pelvis was normal while penile fast sonography revealed an inhomogeneous broad thickening of subcutaneous tissues with increased vascularity on Color-Doppler. Hence, as the penis was further manipulated in order to reduce oedema, retract foreskin and evaluate the glans, clinical parameters rapidly worsened and the patient developed a septic shock with blood pressure falling down, dyspnoea and tachyarrhythmia. He was hospitalized and quickly sent to Intensive Care Unit. During hospitalization, the patient presented with severe hypoxia, acute respiratory distress, marked hypotension and septic shock signs: no impairment of neurological function was present. On general examination it showed bluish colour of the skin at the level of the fingers totally according to intense cyanosis as an expression of severe hypoxia and peripheral vasoconstriction. Blood gas analysis (BGA) both confirmed hypoxia and metabolic acidosis with high lactate levels. Severe leucocytosis, high levels of procalcitonin 


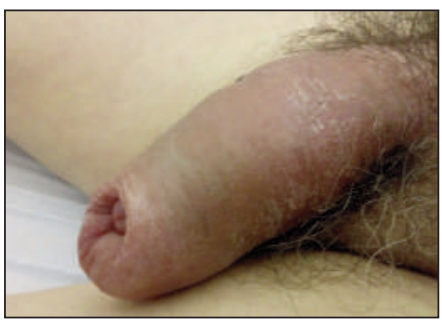

Figure 1.

Uncommon clinical presentation of Fournier's gangrene of the penis.

and C-reactive protein were also confirmed on blood examination. Urine and blood culture sampling were mandatory. A bladder catheter (BC) was placed in order to check diuresis as a marker of septic evolution. Hence, respiratory and haemodynamic support in combination with a broad-spectrum antibiotic therapy were immediately offered according to guidelines for septic shock treatment. Concerning of drugs, we opted for a combination of tazobactam/piperacillin and levofloxacin. However, by persisting severe anuria and hypoxia as a sign of poor response to resuscitation therapy, vasoactive drugs (noradrenaline) were mandatory. Clinical parameters progressively improved while patient started to show a response to drug therapy. Computed tomography (CT) scanning was subsequently performed: it ruled out abscess or any masses altering venous drainage. Urine and blood culture were positive for Enterobacteriaceae (Enterococcus faccalis). Moreover, three days after hospitalization urethral discharge manifested: samples analysis confirmed Enterococcus too. In order to better evaluate penile anatomy and disease extent, Magnetic resonance (MR) was offered. It revealed an abscess at the right cavernosal body with a secundary fistula in the urethra (Figure 2). Furthermore, it appeared as dishomogeneous and hypervascularized with a severe thickening of surrounding soft tissues, as it usually happens in extended panniculitis. Signs of necrosis at the level of Buck's fascia and gas in both cavernosal body and soft tissues were also reported strongly suggesting an uncommon isolated Fournier's disease of the penis. The combined approach "drug therapy/surgery" actually represents the gold standard and may be also combined with vacuum assisted closure (VAC) or hyperbaric oxygen (HBO) therapy. Immediate surgical debridement must be performed as the infection is worsening despite drug therapy: it aims to avoid a fast spread of necrotic tissues and the development towards septic shock. It may be feasible as gangrene clinically manifests but becomes harder in early manifestation since no clear foci of infection are surgically treatable. The main procedure performed is the necrosectomy through the extended and radical demolition of necrotic surgery. Wound clo-

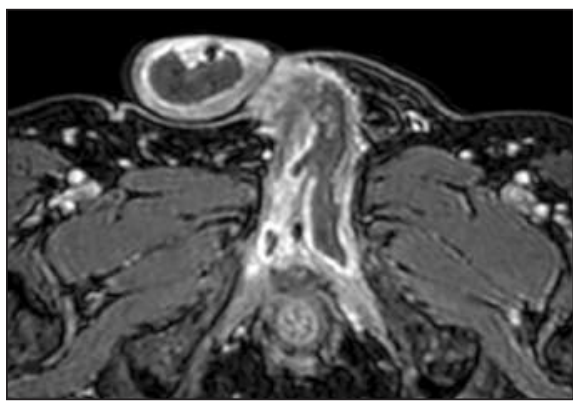

Figure 2

Right cavernosal body involvement and secondary urethral fistula. sure is achieved by different strategies: skin grafting, delayed primary closure and healing by secondary intention. VAC therapy and $\mathrm{HBO}$ may be combined with drugs and surgery since they act as synergic therapies and speed up wound healing. Vacuum-assisted closure has been also proposed but the use is controversial (3). Reconstructive surgery may be considered once the clinical resolution has been obtained. In our patient, considering the clinical absence of gangrene's foci, we opted for a conservative treatment: we performed a urinary diversion by a suprapubic cystostomy and we continued with drug therapy. Subsequently we sent the patient to a reference center for andrology, where they proceeded to the implantation of penile prosthesis.

\section{Conclusions}

Fournier's disease is an aggressive synergistic genitoperineal fasciitis quickly evolving into sepsis and death. Polymicrobial infection actually represents the suggested mechanism. Elderly and patients with multiple comorbidities are at high risk to develop. Clinical presentation may be variable, strongly conditioning diagnosis and treatment: the later diagnosis, the faster evolution, the higher death. Uncommon clinical presentation may lead to a missed diagnosis. Antibiotics and extensive surgical debridement represent the gold standard. Mortality still remains high. Fever and leucocytosis associated to atypical penile swelling should be always evaluated for an uncommon form of Fournier's gangrene.

\section{REFERENCES}

1. Anchi T, Tamura K, Inoue K, et al. Localized Fournier's gangrene of the penis: A case report. Hinyokika Kiyo 2009; 55.

2. Chawla S, Gallop C, Mydlo J. Fournier's gangrene: an analysis of repeated surgical debridement. Eur Urol. 2003; 43:572-575.

3. Yanaral F, Balci C, Ozgor F, et al. Comparison of conventional dressings and vacuum-assisted closure in the wound therapy of Fournier's gangrene. Arch Ital Urol Androl. 2017; 89:208-211.

\author{
Correspondence \\ Valerio Olivieri, MD \\ valerio.olivieri@uniromal.it \\ Division of Urology, Ivrea Civil Hospital (ASL TO4), Ivrea (Turin), Italy \\ Gabriele Ruggiero, MD \\ Daniele Griffa, MD \\ Division of Urology, Ivrea Civil Hospital (ASL TO4), Ivrea (Turin), Italy \\ Danilo Abate, MD \\ Nicoletta Serra, MD \\ Division of Urology, Ciriè Hospital (ASLTO4), Ciriè (Turin), Italy \\ Valentina Fortunati, MD \\ valerio.olivieri@uniromal.it \\ Division of Pathology, Hospital "Policlinico of Tor Vergata", Rome, Italy \\ Flavio Forte, MD \\ flavioforte@hotmail.com \\ Emanuele Corongiu, $M D$ \\ emanuele.corongiu@libero.it \\ Division of Urology, Hospital "Madre Giuseppina Vannini", Rome, Italy
}

